\title{
The Interaction of Waves in the Zero-Pressure Euler Equations with a Coulomb-Like Friction Term
}

\author{
Aiju Wang $\mathbb{D}^{1},{ }^{1}$ Wangxun Yu $\mathbb{D},{ }^{1}$ and Yanyan Zhang $\mathbb{D}^{2}$ \\ ${ }^{1}$ College of Mathematics and Statistics, Xinyang University, Xinyang 464000, China \\ ${ }^{2}$ College of Mathematics and Statistics, Xinyang Normal University, Xinyang 464000, China
}

Correspondence should be addressed to Wangxun Yu; yuwangx@yeah.net

Received 16 October 2021; Revised 7 December 2021; Accepted 29 January 2022; Published 28 February 2022

Academic Editor: Ahmed Zeeshan

Copyright (c) 2022 Aiju Wang et al. This is an open access article distributed under the Creative Commons Attribution License, which permits unrestricted use, distribution, and reproduction in any medium, provided the original work is properly cited.

In this study, the interaction of waves in the zero-pressure Euler equations with a Coulomb-like friction term is considered, which is equivalent to the Riemann problem with three constant initial states for the zero-pressure Euler equations. By solving generalized Rankine-Hugoniot relations under suitable entropy conditions, four different structures of explicit solutions are obtained uniquely, in which the interactions among contact discontinuity, vacuum, and delta shock are presented.

\section{Introduction}

In this study, we investigate the zero-pressure Euler equations with the Coulomb-like friction term:

$$
\left\{\begin{array}{l}
\rho_{t}+(\rho u)_{x}=0, \\
(\rho u)_{t}+\left(\rho u^{2}\right)_{x}=\beta \rho,
\end{array}\right.
$$

where $\rho, u$ are the density and velocity.

It was proposed by Savage and Hutter [1] to describe the granular flow behavior; it can also derive violent discontinuities in shallow flows with large Froude number [2]; the most direct source is the zero-pressure Euler equation (shortened from Euler-Poisson systems) [3].

$$
\left\{\begin{array}{l}
\rho_{t}+(\rho u)_{x}=0 \\
(\rho u)_{t}+\left(\rho u^{2}\right)_{x}=\rho\left(\alpha \Phi_{x}+\beta\right) \\
\Phi_{x x}=\rho
\end{array}\right.
$$

by letting $\alpha=0$.

For system (1), the Riemann problem with two constant initial states was investigated by Shen [4], and the vacuum state and delta shock solution were obtained. The delta shock wave is a new type of discontinuity and is an extension of the traditional shock wave, on which at least one of the state variables develops an extreme concentration. Because more characteristics will enter the discontinuity line, the delta shock wave has greater compressibility. It can be used to describe the process of mass concentration. As is well known, the study of the systems with delta shock wave is very important in applications because it often arises in cosmology, magnetohydrodynamics, and filtration theory [5-7]. For more details about the delta shock waves, we refer to $[8-15]$.

In the study of the delta shock wave, we have to talk about the interaction of waves. In recent years, the discussion for the interaction of waves becomes increasingly active because it plays an important role not only in practical applications but also in the establishment of the general mathematical theory of quasilinear hyperbolic systems. For the research of delta shock waves in zero-pressure gas dynamics, we refer to Yang et al. [16], Shen and Sun [17], and Cheng et al. [18]. See also Cai and Zhang et al. [19, 20] for the zero-pressure gas dynamics with energy conservation law and Zhang and Zhang [21] for the steady zero-pressure adiabatic flow. Moreover, the theoretical results of delta 
shock wave interaction are verified by numerical schemes. It has been deeply studied by many scholars; two delta shock waves overtaking each other are simulated in constant pressure fluid dynamics [22]. For more details about numerical schemes of the interaction of waves, the reader can refer to $[12,14,23]$.

Recently, there are many achievements in the Riemann problem of inhomogeneous conservation law [4, 24-26]. However, little research about the interaction of waves in the inhomogeneous conservation laws has been done; because of this, we study the Riemann problem with three constant initial states,

$$
(\rho, u)(x, 0)= \begin{cases}\left(\rho_{l}, u_{l}\right), & x<x_{01} \\ \left(\rho_{m}, u_{m}\right), & x_{01}<x<x_{02} \\ \left(\rho_{l}, u_{l}\right), & x>x_{02}\end{cases}
$$

for zero-pressure Euler equations with the friction term.

Due to the effect by source term, the characteristics are not straight line and the Riemann solutions are not selfsimilar anymore. So, it is not so easy to get the result of the interaction of waves. Luckily, rewriting system (1) into a conservative form by introducing a new state variable, the delta shock wave is derived by solving generalized Rankine-Hugoniot conditions under suitable entropy conditions, four kinds of solutions of Riemann problems (1) and (3) were established, as given in Section 3. Compared with zeropressure flow, the state variable $u$ changes linearly at the rate $\beta$ with respect to $t$. However, the Riemann solutions of (1) and (3) converge zero-pressure Euler equations as $\beta \longrightarrow 0$.

This study is arranged as follows. In Section 2, we construct some elementary wave solution and singular solution (delta shock wave) about (1). In Section 3, the global solutions for each case are constructed after the interaction of waves, by solving the Riemann problem consisting of (1) and (3). Finally, our conclusions are drawn in Section 3.

\section{Preliminaries}

First, we consider the Riemann problem for (1) with

$$
(\rho, u)(x, 0)= \begin{cases}\left(\rho_{-}, u_{-}\right), & x<0, \\ \left(\rho_{+}, u_{+}\right), & x>0 .\end{cases}
$$

As initial data, by introducing a new state variable, $v=$ $u-\beta t$, the pressureless Euler system with Coulomb-like friction term (1) is rewritten in the conservative form:

$$
\left\{\begin{array}{l}
\rho_{t}+(\rho(v+\beta t))_{x}=0, \\
(\rho v)_{t}+(\rho v(v+\beta t))_{x}=0 .
\end{array}\right.
$$

The eigenvalues and corresponding right eigenvector of system (1) are $\lambda=v+\beta t=u, \vec{r}=(0,1)^{T}$, respectively, which satisfy $\nabla \lambda \cdot \vec{r}=0$. Hence, equations (1) is a linearly degenerated and extremely nonstrict hyperbolic system.

It is easier to verify that besides the constant solution, vacuum state solution (denoted by $\mathrm{Vac}$ ) and two contact discontinuities $J: \omega=u_{-}+\beta t=u_{+}+\beta t$ are included.
It is known from [4] that the solutions of the Riemann problem consisting of (1) and (4) are the following cases:

(1) If $u_{-}<u_{+}$, a vacuum and two contact discontinuities $J_{1}: \omega=u_{-}+\beta t, J_{2}: \omega=u_{+}+\beta t$ are included in the solution:

$$
(\rho, u)(x, t)= \begin{cases}\left(\rho_{-}, u_{-}\right)(x, t), & x<x_{1}(t), \\ \operatorname{Vac}, & x_{1}(t)<x<x_{2}(t), \\ \left(\rho_{+}, u_{+}\right)(x, t), & x(t)>x_{2}(t) .\end{cases}
$$

(2) If $u_{-}>u_{+}$, a delta shock wave delivers from the initial discontinuity point between two constant initial states:

$$
(\rho, u)(x, t)= \begin{cases}\left(\rho_{-}, u_{-}\right)(x, t), & x<x(t) \\ \left(\omega(t) \delta(x-x(t)), u_{\delta}(t)\right), & x=x(t) \\ \left(\rho_{+}, u_{+}\right)(x, t), & x>x(t)\end{cases}
$$

Its curve $x=x(t)$, weight $\omega=\omega(t)$, and the propagation speed $u_{\delta}(t)$ satisfied generalized Rankine-Hugoniot relation,

$$
\left\{\begin{array}{l}
\frac{d x}{d t}=u_{\delta}(t), \\
\frac{d \omega(t)}{d t}=[\rho] u_{\delta}(t)-[\rho u] \\
\frac{d\left(\omega(t) u_{\delta}(t)\right)}{d t}=[\rho u] u_{\delta}(t)-\left[\rho u^{2}\right]+\beta \omega(t),
\end{array}\right.
$$

and the entropy condition

$$
u_{+}+\beta t<u_{\delta}(t)<u_{-}+\beta t,
$$

where $[G]=G_{+}-G_{-}$.

Suppose that $u_{\delta}(t)-\beta t=v_{\delta}$ is a constant; solving (8) with the initial conditions $t=0: x(0)=0, \omega(0)=0$, $u_{\delta}(0)=0$, we can get

$$
\left\{\begin{array}{l}
x(t)=v_{\delta} t+\frac{1}{2} \beta t^{2}, \\
\omega(t)=\sqrt{\rho_{-} \rho_{+}}\left(u_{-}-u_{+}\right) t, \\
u_{\delta}(t)=v_{\delta}+\beta t
\end{array}\right.
$$

where $v_{\delta}=\left(\sqrt{\rho_{-}} u_{-}-\sqrt{\rho_{+}} u_{+}\right)\left(\sqrt{\rho_{-}}-\sqrt{\rho_{+}}\right)$.

Moreover, for more general initial conditions,

$$
\begin{aligned}
t & =0: x(0)=0, \\
\omega(0) & =\omega_{0}, \\
u_{\delta}(0) & =u_{0}\left(u_{+}<u_{0}<u_{-}\right) .
\end{aligned}
$$

Solving (8) with initial condition (11), we can get 


$$
x(t)= \begin{cases}\frac{\omega_{0} u_{0} t-\left[\rho u^{2}\right] / 2 t^{2}}{\omega_{0}-[\rho u] t}+\frac{1}{2} \beta t^{2}, & \rho_{-}=\rho_{+}, \\ \frac{[\rho u] t+\omega(t)-\omega_{0}}{[\rho]}+\frac{1}{2} \beta t^{2}, & \rho_{-} \neq \rho_{+},\end{cases}
$$

$$
\omega(t)=\left(\rho_{-} \rho_{+}\left(u_{-}-u_{+}\right)^{2} t^{2}+2 \omega_{0}\left([\rho] u_{0}-[\rho u]\right) t+\omega_{0}^{2}\right)^{(1 / 2)},
$$

$u_{\delta}(t)=\frac{[\rho u]\left(x-1 / 2 \beta t^{2}\right)-\left[\rho u^{2}\right] t+\omega_{0} u_{0}}{\omega(t)}+\beta t$.

The above delta shock solutions also have the following characteristics.

(i) If $u_{+}<u_{0}<u_{-}, \rho_{-} \neq \rho_{+}$, solution (10) can be obtained by (12)-(14) when $\omega_{0} \longrightarrow 0$

(ii) $u_{+}<u_{\delta}<u_{-}$

\section{Construction of the Solutions to Riemann Problems (1) and (3)}

Discuss the interaction of waves in the zero-pressure Euler equations with a Coulomb-like friction term, which is equivalent to constructing the solutions to the Riemann problems (1) and (3). According to the relation among $u_{l}, u_{m}$, and $u_{r}$, we discuss four cases of solutions as follows.

Case 3.1. $u_{l}>u_{m}>u_{r}$.

If $u_{l}>u_{m}>u_{r}$, two delta shocks $\delta_{1}$ and $\delta_{2}$ deliver from the initial discontinuity points $\left(x_{01}, 0\right)$ and $\left(x_{02}, 0\right)$ separately.

According to Section 2, we get two delta shocks:

$$
\begin{aligned}
& \delta_{1}:\left\{\begin{array}{l}
x(t)=\frac{\sqrt{\rho_{l}} u_{l}-\sqrt{\rho_{m}} u_{m}}{\sqrt{\rho_{l}}-\sqrt{\rho_{m}}} t+\frac{1}{2} \beta t^{2}+x_{01}, \\
\omega_{\delta_{1}}(t)=\sqrt{\rho_{l} \rho_{m}}\left(u_{l}-u_{m}\right) t, \\
u_{\delta_{1}}(t)=\frac{\sqrt{\rho_{l}} u_{l}-\sqrt{\rho_{m}} u_{m}}{\sqrt{\rho_{l}}-\sqrt{\rho_{m}}}+\beta t,
\end{array}\right. \\
& \delta_{2}:\left\{\begin{array}{l}
x(t)=\frac{\sqrt{\rho_{m}} u_{m}-\sqrt{\rho_{r}} u_{r}}{\sqrt{\rho_{m}}-\sqrt{\rho_{r}}} t+\frac{1}{2} \beta t^{2}+x_{02}, \\
\omega_{\delta_{2}}(t)=\sqrt{\rho_{m} \rho_{r}}\left(u_{m}-u_{r}\right) t \\
u_{\delta_{2}}(t)=\frac{\sqrt{\rho_{m}} u_{m}-\sqrt{\rho_{r}} u_{r}}{\sqrt{\rho_{m}}-\sqrt{\rho_{r}}}+\beta t,
\end{array}\right.
\end{aligned}
$$

and all of them satisfy the entropy condition $u_{l}>u_{\delta_{1}}>u_{m}>u_{\delta_{2}}>u_{r}$ simultaneously.

Because the speed of $\delta_{1}$ is faster than the speed of $\delta_{2}$, two delta shocks will impact at a finite time $t^{*}=x_{02}-x_{01} / u_{\delta_{1}}-u_{\delta_{2}}$. Since $u_{l}>u_{r}$, right now, a new
Riemann problem with a new delta shock $\delta: x=x(t)$ solution will be formed, and its velocity $u_{\delta}$ and weight $\omega(t)$ satisfy

$$
t=t^{*}:\left\{\begin{array}{l}
x\left(t^{*}\right)=x_{1}\left(t^{*}\right)=x_{2}\left(t^{*}\right), \\
\omega\left(t^{*}\right)=\omega_{\delta_{1}}\left(t^{*}\right)=\omega_{\delta_{2}}\left(t^{*}\right), \\
u_{\delta}\left(t^{*}\right)=\frac{\omega_{\delta_{1}}\left(t^{*}\right) u_{\delta_{1}}+\omega_{\delta_{2}}\left(t^{*}\right) u_{\delta_{2}}}{\omega_{\delta_{1}}\left(t^{*}\right) u_{\delta_{1}}+\omega_{\delta_{2}}\left(t^{*}\right) u_{\delta_{2}}}=u_{\delta} .
\end{array}\right.
$$

According to the conservation of mass and momentum at $t=t^{*}$, it is easy to check that $u_{\delta_{1}}>u_{\delta}^{*}>u_{\delta_{2}}$. In the same way as above, by solving (8) with initial condition (17), we get the locus, velocity, and mass of the delta shock $\delta:\left(x(t), \omega(t), u_{\delta}(t)\right),\left(t_{2} \leq t<+\infty\right)$. The details are spared. To sum up, in the case that $u_{l}>u_{m}>u_{r}$, we come to the following conclusion: the two delta shocks must collide with each other at a finite time and merge into a new delta shock, as shown in Figure 1. We can express this fact as

$$
\delta \delta \longrightarrow \delta \text {. }
$$

Case 3.2. $u_{l}>u_{r}>u_{m}$. (When $u_{m}>u_{l}>u_{r}$, it has the same structure as the solution).

Since $u_{l}>u_{m}$, a delta shock $\delta_{1}$ delivers from the initial discontinuity point $\left(x_{01}, 0\right)$, whose trajectory, mass, and velocity can be expressed as in (15). Since $u_{m}<u_{r}$, the vacuum and two contact discontinuities $J_{1}: u=u_{m}+\beta t$, $J_{2}: u=u_{r}+\beta t$ emit from the discontinuity point $\left(x_{02}, 0\right)$, as shown in Figure 2.

Based on the entropy condition, we have $u_{l}>u_{\delta_{1}}>u_{m}$. The delta shock $\delta_{1}$ meets the contact discontinuity $J_{1}$ at a finite time $t_{1}=x_{02}-x_{01} / u_{\delta_{1}}-u_{m}$. Note that

$$
\begin{aligned}
& x_{1}=x\left(t_{1}\right), \\
& \omega_{1}=\omega_{\delta 1}\left(t_{1}\right), \\
& u_{\delta_{1}}=u_{\delta}\left(t_{1}\right) .
\end{aligned}
$$

After time $t_{1}$, a new Riemann problem solvable with a delta shock wave solution is formed and denoted as $\delta_{2}$, which satisfy the generalized Rankine-Hugoniot relation as follows:

$$
\left\{\begin{array}{l}
\frac{d x}{d t}=u_{\delta}, \\
\frac{d \omega}{d t}=\rho_{l} u_{l}-\rho_{l} u_{\delta}+\rho_{l} \beta t, \\
\frac{d\left(\omega u_{\delta}\right)}{d t}=\rho_{l} u_{l}^{2}+2 \rho_{l} u_{l} t+\rho_{l} \beta^{2} t^{2}-\left(\rho_{l} u_{l}+\rho_{l} \beta t\right) u_{\delta}(t)+\beta \omega .
\end{array}\right.
$$

Use the same method as above; solving (20) with initial condition (19), we get the locus, velocity, and mass of the delta shock $\delta_{2}$ : 


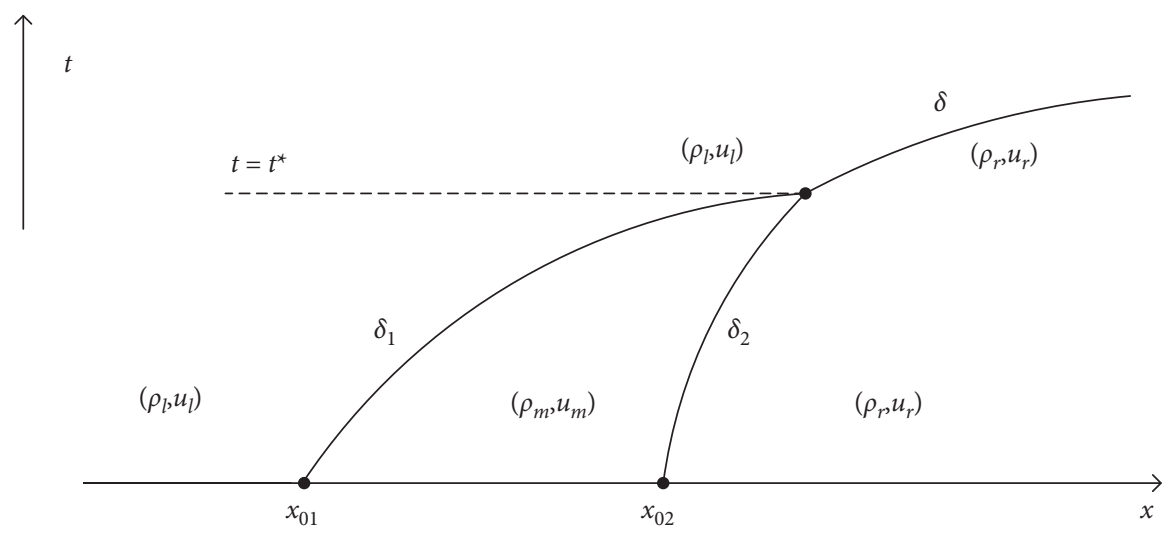

FIGURE 1: The collision of two delta shock waves.

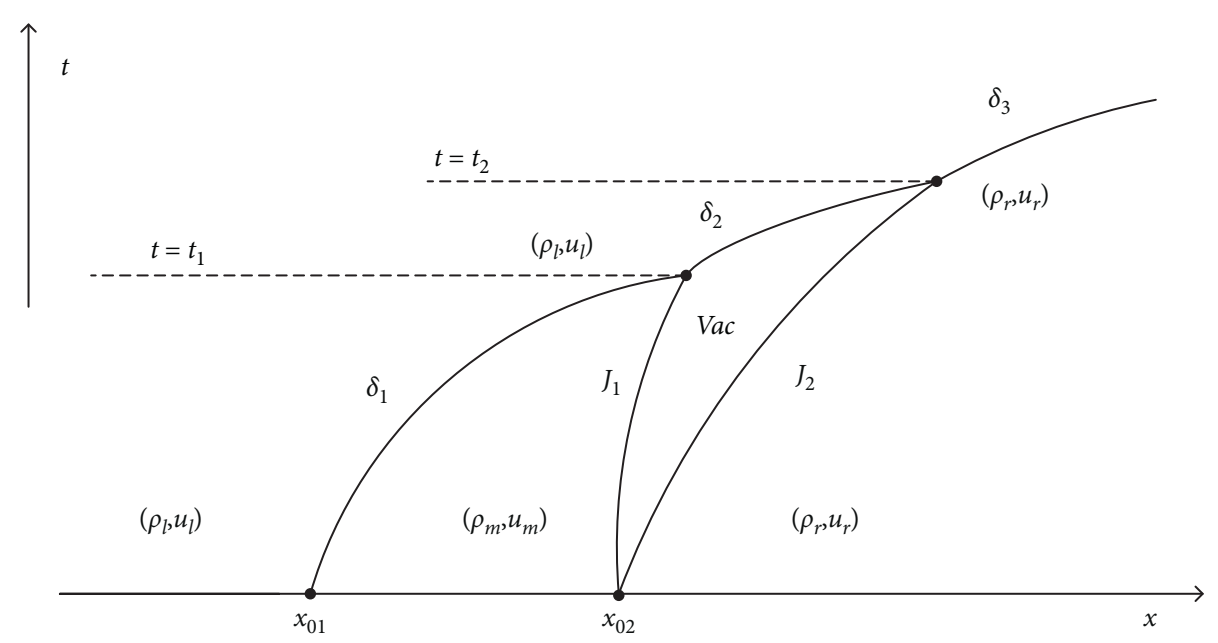

FIGURE 2: The delta shock overtakes the vacuum completely.

$\left\{\begin{array}{l}x=x_{2}(t)=\frac{\omega_{1}+\rho_{l} u_{l} t-\omega_{2}(t)}{\rho_{l}}+\frac{1}{2} \beta t^{2}+x_{1}, \\ \omega=\omega_{2}(t)=\left(\omega_{1}^{2}+2 \omega_{1} \rho_{l}\left(u_{l}-u_{\delta_{1}}\right) t\right)^{(1 / 2)}, \\ u_{\delta_{2}}(t)=\frac{\omega_{1} u_{\delta_{1}}+\rho_{l} u_{l}^{2} t-\rho_{l} u_{l}\left(x_{2}(t)-1 / 2 \beta t^{2}\right)}{\omega_{2}(t)}+\beta t .\end{array}\right.$

Since $\lim _{t \rightarrow \infty}\left(u_{\delta_{2}}(t)-\beta t\right)=u_{l}>u_{r}$, the delta shock $x=x_{2}(t)$ will overtake the contact discontinuity $J_{2}: u=u_{r}+\beta t$ starting at a finite time $t_{2}$, that is, $x\left(t_{2}\right)=u_{r} t_{2}+1 / 2 \beta t_{2}^{2}+x_{02}$. At this moment, since $u_{l}>u_{r}$, a new Riemann problem solvable with a delta shock wave solution is formed, whose trajectory $x(t)$, weight $\omega(t)$, and velocity $u_{\delta}$ satisfy

$$
\begin{aligned}
t & =t_{2}: x_{2}=x\left(t_{2}\right), \\
\omega_{2} & =\omega_{2}\left(t_{2}\right), \\
u_{\delta_{2}} & =u_{\delta_{2}}\left(t_{2}\right) .
\end{aligned}
$$

It is easy to check that $u_{r}+\beta t<u_{\delta_{2}}<u_{l}+\beta t$. In the same way as above, solving (9) with initial data (22), we obtain $\delta_{3}:\left(x(t), \omega(t), u_{\delta}(t)\right)\left(t_{2} \leq t<+\infty\right)$. The details are spared. To sum up, in the case that $u_{l}>u_{r}>u_{m}$, the delta shock collides two contact discontinuities and overtakes the vacuum completely in bounded time (Figure 2). We can express this fact as

$$
\delta J V a c J \longrightarrow \delta
$$

Case 3.3. $u_{r}>u_{l}>u_{m}$. (When $u_{m}>u_{r}>u_{l}$, it has the same structure as the solution).

The same as in Case 3.2, a delta shock delivers from the initial discontinuity point $\left(x_{01}, 0\right)$, two contact discontinuities, and a vacuum emits from $\left(x_{02}, 0\right)$, as shown in Figure 2. The delta shock $\delta_{1}$ collides with $J_{1}$ starting at time $t_{1}$ and overtakes the vacuum. The procession is the same as that in Case 3.2, and (20) and (21) are tenable. But since $\lim _{t \rightarrow \infty}\left(u_{\delta_{2}}(t)-\beta t\right)=u_{l}<u_{r}, \quad u_{\delta_{2}}(t)<u_{r}+\beta t$, it shows that the delta shock $\delta_{2}$ never collides with $J_{2}$. That is different from the structure in Case 3.2; under the condition in Case 3.3, the delta shock has not overtaken the vacuum completely (Figure 3 ). We can express this fact as

$$
\delta J V a c J \longrightarrow \delta V a c J
$$




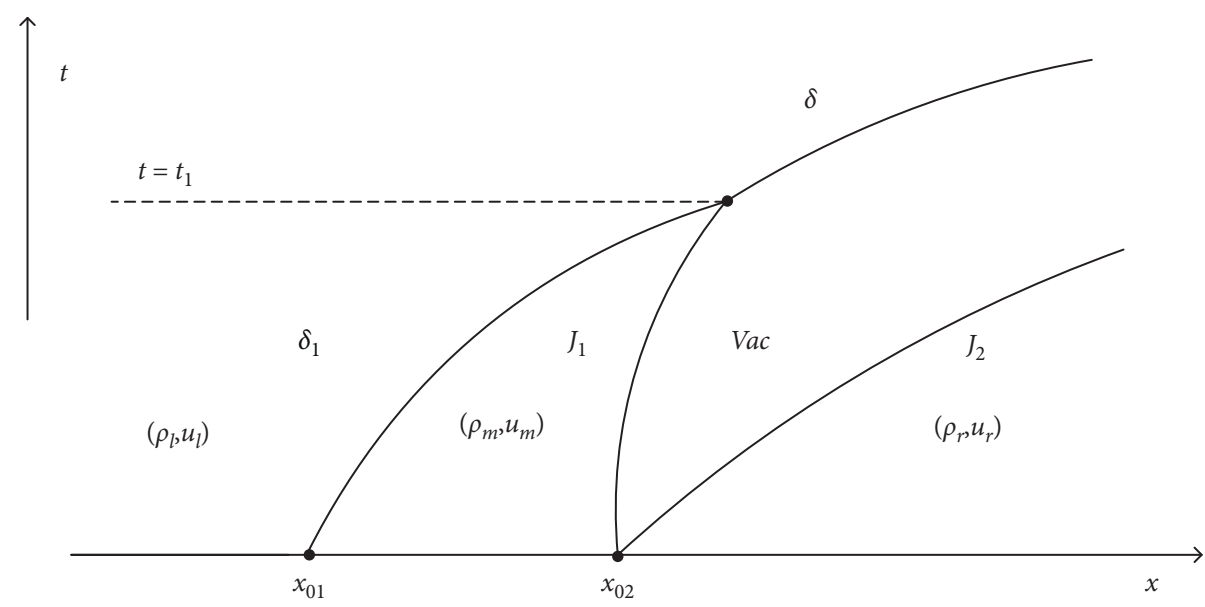

FIgURE 3: The delta shock does not overtake the vacuum completely.

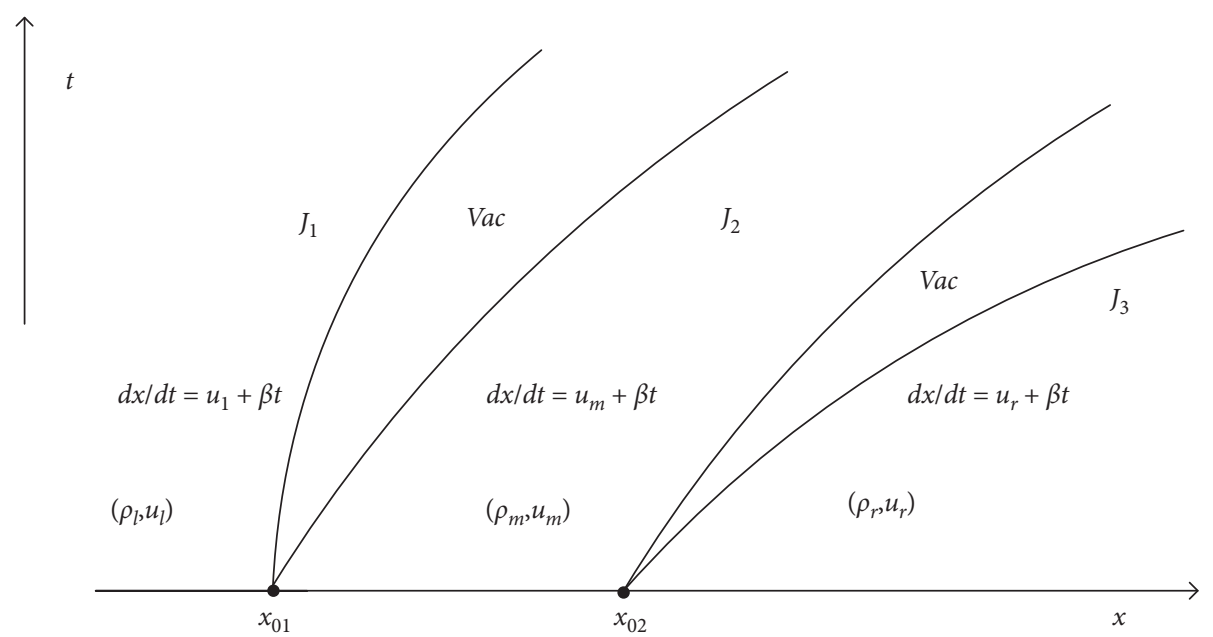

Figure 4: No collision status.

Case 3.4. $u_{r}>u_{m}>u_{l}$.

If $u_{r}>u_{m}>u_{l}$, there is no collision or overtake of waves. We can express the Riemann solution of systems (1) and (3) as

$$
(\rho, u)(t, x)= \begin{cases}\left(\rho_{l}, u_{l}+\beta t\right), & x \leq u_{l} t+\frac{1}{2} \beta t^{2}+x_{01} \\ \text { Vac, } & u_{l} t \leq x-x_{01}-\frac{1}{2} \beta t^{2} \leq u_{m} t ; \\ \left(\rho_{m}, u_{m}+\beta t\right), & u_{l} t+x_{01} \leq x-\frac{1}{2} \beta t^{2} \leq u_{m} t+x_{02} \\ \operatorname{Vac}, & u_{m} t \leq x-x_{02}-\frac{1}{2} \beta t^{2} \leq u_{r} t \\ \left(\rho_{r}, u_{r}+\beta t\right), & x \geq u_{r} t+\frac{1}{2} \beta t^{2}+x_{02} .\end{cases}
$$

We call it a collisionless solution, as shown in Figure 4.

\section{Conclusion}

Through the analysis above, we have constructed the Riemann solutions for zero-pressure Euler equations with the friction term. In particular, elementary wave solution and singular solution (delta shock wave) have been discovered under certain situations. We find that the characteristic lines and the delta shock wave discontinuity are curved under the influence of friction terms. Compared with previous results on generalized zero-pressure gas dynamics [16], there are two interesting new phenomena. The Riemann solutions of (1) and (3) are not self-similar anymore, and the state variable $u$ changes linearly at the rate $\beta$ with respect to $t$. Luckily, four kinds of solutions were obtained by laborious calculations, including collisions of two shocks, shock overtaking vacuum, and two vacuum pursuits, showing the interaction of waves clearly. The result is similar to zeropressure flow. It has proved the correctness of the theory from another side, since the Riemann solutions of (1) and (3) converge zero-pressure Euler equations as $\beta \longrightarrow 0$, namely, the friction term vanishes. Moreover, it also provides a 
theoretical guarantee for the subsequent numerical simulation.

\section{Data Availability}

No data were used to support this study.

\section{Conflicts of Interest}

The authors declare that they have no conflicts of interest.

\section{References}

[1] S. B. Savage and K. Hutter, "The motion of a finite mass of granular material down a rough incline," Journal of Fluid Mechanics, vol. 199, pp. 177-215, 1989.

[2] C. M. Edwards, S. D. Howison, H. Ockendon, and J. R. Ockendon, "Non-classical shallow water flows," IMA Journal of Applied Mathematics, vol. 73, pp. 137-157, 2008.

[3] T. Nguyen and A. Tudorascu, "Pressureless Euler/EulerPoisson systems via adhesion dynamics and scalar conservation laws," SIAM Journal on Mathematical Analysis, vol. 40, no. 2, pp. 754-775, 2008.

[4] C. Shen, "The Riemann problem for the Chaplygin gas equations with a source term," ZAMM - Journal of Applied Mathematics and Mechanics/Zeitschrift für Angewandte Mathematik und Mechanik, vol. 96, no. 6, pp. 681-695, 2016.

[5] B. T. Hayfs and P. G. Lefloch, "Measure solutions to a strictly hyperbolic system of conservation laws," Nonlinearity, vol. 9, no. 6, pp. 1547-1563, 1996.

[6] E. Weinan, Y. G. Rykov, and Y. G. Sinai, "Generalized variational principles, global weak solutions and behavior with random initial data for systems of conservation laws arising in adhesion particle dynamics," Communications in Mathematical Physics, vol. 177, no. 2, pp. 349-380, 1996.

[7] V. G. Danilov and V. M. Shelkovich, "Dynamics of propagation and interaction of $\delta$-shock waves in conservation law systems," Journal of Differential Equations, vol. 211, no. 2, pp. 333-381, 2005.

[8] B. L. Keyfitz and H. C. Kranzer, "Spaces of weighted measures for conservation laws with singular shock solutions," Journal of Differential Equations, vol. 118, no. 2, pp. 420-451, 1995.

[9] D. C. Tan and T. Zhang, "Two-Dimensional Riemann problem for a hyperbolic system of nonlinear conservation laws," Journal of Differential Equations, vol. 111, no. 2, pp. 203-254, 1994.

[10] D. C. Tan, T. Zhang, T. Chang, and Y. X. Zheng, "Delta-shock waves as limits of vanishing viscosity for hyperbolic systems of conservation laws," Journal of Differential Equations, vol. 112, no. 1, pp. 1-32, 1994.

[11] F. Huang and Z. Wang, "Well-posedness for pressureless flow," Communications in Mathematical Physics, vol. 222, pp. 117-146, 2001.

[12] H. Yang, "Riemann problems for a class of coupled hyperbolic systems of conservation laws," Journal of Differential Equations, vol. 159, no. 2, pp. 447-484, 1999.

[13] H. Yang and Y. Zhang, "New developments of delta shock waves and its applications in systems of conservation laws," Journal of Differential Equations, vol. 252, pp. 5951-5993, 2012.

[14] H. Yang and Y. Zhang, "Delta shock waves with Dirac delta function in both components for systems of conservation laws," Journal of Differential Equations, vol. 257, no. 12, pp. 4369-4402, 2014.
[15] W. Sheng and T. Zhang, "The Riemann problem for transportation equation in gas dynamics," Memoirs of the American Mathematical Society, vol. 137, pp. 1-77, 1999.

[16] H. Yang, R. Hu, and Y. Sun, "The Riemann problem with three constant initial states for one-dimensional zero-pressure gas dynamics," J. Southeast Asian Bull Math, vol. 33, pp. 179-187, 2009.

[17] C. Shen and M. Sun, "Interactions of delta shock waves for the transport equations with split delta functions," Journal of Mathematical Analysis and Applications, vol. 351, no. 2, pp. 747-755, 2009.

[18] H. Cheng, W. Liu, and H. Yang, "Two-dimensional Riemann problems for zero-pressure gas dynamics with three constant states," Journal of Mathematical Analysis and Applications, vol. 343, pp. 127-140, 2008.

[19] W. Cai and Y. Zhang, "Interactions of delta shock waves for zero-pressure gas dynamics with energy conservation law," Advances in Mathematical Physics, vol. 2016, Article ID 1783689, 12 pages, 2016.

[20] Y. Zhang and Y. Zhang, "Generalized plane delta shock waves for the $\mathrm{n}$-dimensional zero-pressure gas dynamics with energy conservation law," Indian Journal of Pure and Applied Mathematics, vol. 50, pp. 1067-1086, 2019.

[21] Y. Zhang and Y. Zhang, "The Riemann problem and interaction of waves in two-dimensional steady zero-pressure adiabatic flow," International Journal of Non-linear Mechanics, vol. 104, pp. 100-108, 2018.

[22] Y. Zhang and Y. an Zhang, "Interactions of delta shock waves for the equations of constant pressure fluid dynamics," Differential Equations \& Applications, vol. 13, no. 1, pp. 63-84, 2021.

[23] E. F. Toro, Riemann Solvers and Numerical Methods for Fluid Dynamics, Springer, Berlin, 1999.

[24] C. Shen, "The Riemann problem for the zero-pressure Euler equations with the Coulomb-like friction term," IMA Journal of Applied Mathematics, vol. 81, pp. 76-99, 2016.

[25] M. Sun, "The exact Riemann solutions to the generalized Chaplygin gas equations with friction," Communications in Nonlinear Science and Numerical Simulation, vol. 36, pp. 342-353, 2016.

[26] Y. Zhang, Y. Zhang, and Y. Zhang, "Riemann problems for a class of coupled hyperbolic systems of conservation laws with a source term," Communications on Pure and Applied Analysis, vol. 18, no. 3, pp. 1523-1545, 2019. 